Honam Mathematical J. 36 (2014), No. 1, pp. 187-198

http://dx.doi.org/10.5831/HMJ.2014.36.1.187

\title{
THE CONSTRUCTION OF FUZZY IDEMPOTENT ZERO PATTERNS BY A PROGRAM
}

\author{
Se Won Park and Chul Kang*
}

\begin{abstract}
The fuzzy idempotent matrices are important in various applications and have many interesting properties. Using the upper diagonal completion process, we have the zero patterns of fuzzy idempotent matrix, that is, Boolean idempotent matrices. And we give the construction of all fuzzy idempotent matrices for some dimention.
\end{abstract}

\section{Introduction}

The theory of a fuzzy matrix is very useful in the discussion of fuzzy relations. We can represent basic propositions of the theory of fuzzy relations in terms of matrix operations. Futhermore we can deal with the fuzzy relations in the matrix form. In the study of the theory of a fuzzy matrix, a canonical form of some fuzzy matrices has received increasing attention. For example, Kim and Roush $[1,2]$ studies the canonical form of an idempotent matrix in 1980 and 1981. Hashimoto [3] studied the canonical form of a transitive matrix in 1983. Their main result is:

For an idempotent (transitive, strongly transitive) fuzzy matrix $R$ there exists a permutation matrix $P$ such that $T=\left[t_{i j}\right]=P \times R \times P^{T}$ satisfies $t_{i j} \leq t_{j i}$, for $i>j$.

The above $T$ was called the canonical form of an idempotent (transitive, strongly transitive) fuzzy matrix by these authors. Clealy, the study of this kind of canonical forms is important to develop the theory of a fuzzy matrix.

Received January 10, 2014. Accepted February 15, 2014.

2010 Mathematics Subject Classification. 11A11.

Key words and phrases. fuzzy idempotent matrix, zero patterns, Boolean matrix.

${ }^{*}$ Corresponding author 
We define some operations for fuzzy matrices whose elements are in the unit interval $[0,1]$. First for $x, y \in[0,1]$ we define $x \vee y$, and $x \wedge y$ as follows:

$$
\begin{aligned}
& x \vee y=\max (x, y) \\
& x \wedge y=\min (x, y) .
\end{aligned}
$$

Next we define the following matrix operations for $n \times n$ fuzzy matrices $R=\left[r_{i j}\right]$ and $S=\left[s_{i j}\right]$ :

$$
\begin{aligned}
& \cdot R \vee S=\left[r_{i j} \vee s_{i j}\right] ; \\
& \cdot R \wedge S=\left[r_{i j} \wedge s_{i j}\right] ; \\
& \cdot R \times S=\left[\left(r_{i 1} \wedge s_{1 j}\right) \vee\left(r_{i 2} \wedge s_{2 j}\right) \vee \cdots \vee\left(r_{i n} \wedge s_{n j}\right)\right] ; \\
& \cdot R^{T}=\left[r_{j i}\right] \quad(\text { the transpose of } R) ; \\
& \cdot R \leq S \quad \text { if and only if } \quad r_{i j} \leq s_{i j} \quad \text { for all } i, j .
\end{aligned}
$$

We define some special kinds of fuzzy matrices. A fuzzy matrix $R$ is said to be:

$$
\begin{aligned}
& \text { - (max-min) transitive if } R^{2} \leq R \text {; } \\
& \text { - idempotent if } R^{2}=R
\end{aligned}
$$

Accordingly, any fuzzy idempotent matrix is transitive.

\section{The Properties of Fuzzy Idempotent Matrices}

First we are examine some basic properties of idempotent fuzzy matrices. They are useful in the following discussion. We know that all $1 \times 1$ fuzzy matrices are idempotent. Hence, in this paper, we deal only with square fuzzy matrix with dimension $n, n \geq 2$. Let $F_{I}$ be the set of all idempotent fuzzy matrices.

Lemma 2.1. The set of all idempotent fuzzy matrices, $F_{I}$, is closed under the following operations:

(i) permutation similarity; and

(ii) transposition

Lemma 2.2. (In [3]) For an idempotent marix $R$ there exists a permutation matrix $P$ such that $T=\left[t_{i j}\right]=P \times R \times P^{T}$ satisfies $t_{i j} \geq t_{j i}$, for $i>j$.

We know the fact that the condition $t_{i j} \geq t_{j i}$ in the above Lemma is changeable to $t_{i j} \leq t_{j i}$ by Lemma 2.1-(ii). Thus we will use the later one from now on. 
Definition 2.3. Let $A$ be a fuzzy matrix. For some nonzero entry a of $A, Z(a)=\left[z_{i j}\right]$ is defined by

$$
z_{i j}=\left\{\begin{array}{l}
1 \text { if } 0<a_{i j} \leq a \\
0 \text { if otherwise }
\end{array}\right.
$$

$Z(a)=\left[z_{i j}\right]$ is called a zero pattern of $A$.

Example 2.4. If a fuzzy matrix

$$
A=\left[\begin{array}{ccc}
0.2 & 0.7 & 1 \\
0.7 & 0 & 0.2 \\
0.3 & 1 & 0
\end{array}\right]
$$

then all its zero patterns are

$$
\begin{aligned}
& Z(0.2)=\left[\begin{array}{lll}
1 & 0 & 0 \\
0 & 0 & 1 \\
0 & 0 & 0
\end{array}\right], \quad Z(0.3)=\left[\begin{array}{lll}
1 & 0 & 0 \\
0 & 0 & 1 \\
1 & 0 & 0
\end{array}\right], \\
& Z(0.7)=\left[\begin{array}{lll}
1 & 1 & 0 \\
1 & 0 & 1 \\
1 & 0 & 0
\end{array}\right], \text { and } Z(1)=\left[\begin{array}{lll}
1 & 1 & 1 \\
1 & 0 & 1 \\
1 & 1 & 0
\end{array}\right] .
\end{aligned}
$$

Lemma 2.5. (In [1]) A fuzzy matrix is idempotent if and only if all its zero patterns are idempotent.

By the above Lemma 2.5, we examine the properties of $(0,1)$-fuzzy matrices and obtain a theorem and canonical form of the $(0,1)$-fuzzy matrices. Thus we will be able to characterize the structure of the set of all fuzzy idempotent matrices, $F_{I}$. Let $B_{I}$ be the set of all idempotent $(0,1)$-fuzzy matrices. In fact, $B_{I}$ is the set of Boolean idempotent matrices. In this paper, the set of numbers $\{1,2,3, \cdots n-1, n\}$ will be denoted by $N$.

Remark 2.6. For an $n \times n$ fuzzy matrix $A, A$ is in $B_{I}$ and $a_{i j}=1$ if and only if there exists a $k \in N$ such that $a_{i k}=1=a_{k j}$. This statement follows from the fact that since $A=\left[a_{i j}\right]$ is idempotent, we must have that $a_{i j}=\sum_{k=1}^{k=n} a_{i k} a_{k j}$ for all $i, j \in N$. Another way of writing this is to observe that $A \in B_{I}$ if and only if for all $i R_{i}=\sum_{j} R_{j}$ where $j=\left\{k \mid e_{k} \leq R_{i}\right\}$ and $C_{i}=\sum_{j} C_{j}$ where $j=\left\{k \mid e^{k} \leq C_{i}\right\}, e_{k}\left(e^{k}\right)$ is an row(column) vector in the standard basis. 


\section{The $(0,1)$-fuzzy Idempotent Matrices}

To simplify notation, in the remainder of this paper we let the index set $\{1,2, \cdots, n\}$ be represented by $N$. A matrix $A$ of order $n \geq 2$ is said to be reducible if there exists a permutation matrix $P$ such that

$$
P A P^{T}=\left(\begin{array}{ll}
B & C \\
O & D
\end{array}\right)
$$

where $B$ and $D$ are square matrices of order at least one. Otherwise $A$ is called irreducible.

Lemma 3.1. Let $A=\left[a_{i j}\right]$ be an $n \times n(2 \leq n)$ fuzzy idempotent matrix. If $a_{i j}=0$ for some $i$ and $j$ in $N$, then each product $a_{i k} a_{k j}=0$ for all $k$ in $N$.

Proof. It is an immediate consequence of the fuzzy matrix product.

An $n \times n(2 \leq n)$ fuzzy matrix $A=\left[a_{i j}\right]$ is said to be graphicaltransitive if $a_{i k} \neq 0$ and $a_{k j} \neq 0$ imply that $a_{i j} \neq 0$ for some $i$ and $j$ in $N$.

Lemma 3.2. (In [5]) If an $n \times n$ Boolean matrix $A$ is idempotent, then $A$ is graphical-transitive.

Lemma 3.3. (In [5]) If $A=\left[a_{i j}\right]$ be an $n \times n$ irreducible Boolean idempotent matrix, then $A$ is entrywise nonzero.

From Lemma 3.3, we know that an irreducible $n \times n$ Boolean idempotent matrix is entrywise nonzero and use only 1 or 0 entries in $B_{I}$. Consequently an irreducible $(0,1)$-fuzzy idempotent matrix may be taken to be an entrywise 1 matrix, that is, $J_{n}=\left[1_{i j}\right]$. We state this formally as follows:

Theorem 3.4. If $A=\left[a_{i j}\right]$ is an $n \times n$ irreducible $(0,1)$-fuzzy idempotent matrix, that is, $A$ is irreducible in $B_{I}$, then it is entrywise 1 (up to equivalences, as stated in Lemma 2.1).

Let an $n \times n$ matrix $A$ be given. Then either $A$ is irreducible or there exists a permutation matrix $P$ such that

$$
P^{T} A P=\left[\begin{array}{ccc}
A_{1} & & * \\
& \ddots & \\
0 & & A_{k}
\end{array}\right]
$$


in which $A_{i}$ is either irreducible or is $1 \times 1$ zero matrix, $i=1, \cdots, k$. This is called the Frobenious normal form of $A$. If $A$ is a reducible fuzzy idempotent matrix in Frobenious normal form, then it is clear that each irreducible diagonal block of $A$ is fuzzy idempotent matrix. In the remainder of this paper, we use the results of above section, and assume that each nonzero irreducible diagonal block $A_{i i}$ of $A$ is entrywise 1 , that is, $A_{i i}=J_{i}$ which are same size. Futher, in terms of block multiplication where $P=A^{2}$,

$$
P_{i j}=A_{i i} A_{i j}+A_{i, i+1} A_{i+1, j}+\cdots+A_{i j} A_{j j}=A_{i j} .
$$

The key question here is: What are the possible zero patterns of the offdiagonal blocks $A_{i j}$ so that the above equation is satisfied? The remainder of this paper is dedicated to answering this question and all nonzero matrix(or block) means entrywise 1 matrix, that is, $J=\left[1_{i j}\right]$.

Lemma 3.5. (In [5]) Let $A=\left[a_{i j}\right]$ be an $n \times n$ reducible Boolean idempotent matrix. If $A_{i i}$ and $A_{j j}$ are nonzero $n_{i} \times n_{i}$ and $n_{j} \times n_{j}$ matrices, respectively, and if $n_{i} \times n_{j}$ matrix $A_{i j}$ contains a zero entry, then $A_{i j}$ is a 0-block.

Lemma 3.6. (In [5]) If $A_{i i}$ and $A_{j j}$ are respectively $n_{i} \times n_{i}$ and $n_{j} \times n_{j}$ entrywise nonzero Boolean matrices, and $A_{i j}$ is an $n_{i} \times n_{j}$ entrywise nonzero matrix, then

(i) $A_{i i} A_{i j}$ is defined if and only if each column $A_{i j}$ contains only 1's, and

(ii) $A_{i j} A_{j j}$ is defined if and only if each row $A_{i j}$ contains only 1's.

Combining Lemma 3.5 and 3.6, we obtain the following lemma.

Lemma 3.7. (In [5]) If $A$ is an $n \times n$ reducible Boolean matrix such that $A_{i i}$ and $A_{j j}$ are entrywise nonzero diagonal blocks, then $A$ is Boolean idempotent only if the entries of $A_{i j}$ are obtained as follows:

(i) $A_{i j}$ contains only 1's, or

(ii) $A_{i j}$ is 0-block.

Lemma 3.8. (In [5]) Let $A$ is an $n \times n$ reducible Boolean idempotent matrix where $A_{i i}$ is an $n_{i} \times n_{i}$ entrywise nonzero diagonal block and $A_{j j}$ is a 0-block. If $A_{i j}$ contains a 0 -entry, then $A_{i j}$ contains a 0-column.

Since a Boolean matrix is idempotent only if each off-diagonal block $P_{i j}$ is defined, we obtain the following:

Lemma 3.9. (In [5]) Let $A$ be an $n \times n$ reducible Boolean idempotent matrix. If $A_{i i}$ is entrywise nonzero and $A_{j j}$ is 0-block, then $A$ is Boolean idempotent only if $A_{i j}=0$ or $A_{i j}$ contains only 1's. 
We should remark that if $A_{i i}$ is a 0-block and $A_{j j}$ is an entrywise nonzero matrix, then we can state results for $A_{i j}$ analogous to those given in Lemma 3.8 and 3.9. In the remainder of this paper, we refer to the latter results as Lemma 3.8 (ii) and 3.9 (ii).

If $A$ is an $m \times m$ block reducible matrix, then the off-diagonal blocks $A_{i, i+k}$ lie on the $k$-th superdiagonal for all $k=1,2, \cdots, m-1$. Due to the triangular structure of $A$, each $P_{i, i+k}$ in the product matrix $P=A^{2}$, as described in the following:

Algorithm 3.10. (In [5]) (The upper diagonal completion process) Let $A=\left[A_{i j}\right]$ be an $m \times m$ reducible, partial block Boolean idempotent matrix in modified Frobenius normal form. Determinied the entry of each off-diagonal block as follows:

(i) Start with 1-st superdiagonal. Determine the entries of each offdiagonal block $A_{i, i+1}$ using Lemma 3.7 if $A_{i i}$ and $A_{i+1, i+1}$ are entrywise nonzero , Lemma 3.9 for each diagonal block of $A_{i+1, i+1}$ if $A_{i i}$ is an entrywise nonzero and $A_{i+1, i+1}$ is a 0 -block, or Lemma 3.9 (ii) for each diagonal block of $A_{i i}$ so that $A_{i-1, i} A_{i, i+1}$ is unambiguously defined if $A_{i i}$ is a 0 -block and $A_{i+1, i+1}$ is an entrywise nonzero fuzzy matrix, Move up to the next diagonal (if there is one).

(ii) For each unsecified entry $A_{i, i+k}$ on the $k$-th superdiagonal, $k=$ $2,3, \cdots, m-1$, if $P_{i, i+k}=A_{i i} A_{i, i+k}+A_{i, i+k} A_{i+k, i+k}$ use step (i) with $i+k$ replacing it, otherwise let $A_{i, i+k}=A_{i, i+1} A_{i+1, i+k}$. When all blocks are specified on this diagonal, move up to the next diagonal, if there is one, increase $k$ by 1 for all $k=2,3, \cdots, m-2$, and repeat (ii).

Example 3.11. Let $A$ is a $(0,1)$-fuzzy idempotent matrix. Then

$$
A=\left[\begin{array}{lllllllll}
1 & 1 & 1 & * & * & * & * & * & * \\
1 & 1 & 1 & * & * & * & * & * & * \\
1 & 1 & 1 & * & * & * & * & * & * \\
0 & 0 & 0 & 0 & 0 & * & * & ? & ? \\
0 & 0 & 0 & 0 & 0 & * & * & ? & ? \\
0 & 0 & 0 & 0 & 0 & 1 & 1 & * & * \\
0 & 0 & 0 & 0 & 0 & 1 & 1 & * & * \\
0 & 0 & 0 & 0 & 0 & 0 & 0 & 0 & 0 \\
0 & 0 & 0 & 0 & 0 & 0 & 0 & 0 & 0
\end{array}\right]
$$

where $*$ is 0 or 1 and ? is determined by the specified number $*$ that is on the lower superdiagonal than it.

Lemma 3.12. (In [5]) Let $A$ is an $n \times n$ reducible Boolean idempotent matrix in modified Frobenious normal form, each of whose diagonal 
blocks is entrywise nonzero or 0-block. Then $A$ is fuzzy idempotent only if each off-diagonal block $A_{i j}$ is obtained using Algorithm 3.10.

Lemma 3.13. (In [5]) A reducible Boolean matrix $A$, in modified Frobenius normal form. each of whose nonzero diagonal block is entrywise 1, is idempotent if and only if the entry of each off-diagonal block is obtained using the upper diagonal completion process.

Since the set of Boolean idempotent matrices is same as the zero patterns of the fuzzy idempotent matrix, we have the following corollary;

Corollary 3.14. A reducible zero patterns of the fuzzy matrix $A$, in modified Frobenius normal form. each of whose nonzero diagonal block is entrywise 1, is idempotent if and only if the entry of each off-diagonal block is obtained using the upper diagonal completion process.

\section{The Construction of Fuzzy Idempotent zero patterns}

In this section, using the condition and properties in the above sections we give all zero patterns and fuzzy idempotent matrix from $n=2$ to $n=5$ by a program that construct fuzzy idempotent zero patterns That is, an $n \times n(0,1)$-fuzzy matrix as $n^{2}$-binary digite form.

[Case, $n=2]$ There are all zero patterns of fuzzy idempotent except the zero matrix as follows;

$$
\begin{aligned}
& A(1)=\left[\begin{array}{ll}
1 & 0 \\
0 & 0
\end{array}\right] \quad A(8)=\left[\begin{array}{ll}
0 & 0 \\
0 & 1
\end{array}\right] \quad A(3)=\left[\begin{array}{ll}
1 & 1 \\
0 & 0
\end{array}\right] \quad A(5)=\left[\begin{array}{ll}
1 & 0 \\
1 & 0
\end{array}\right] \\
& A(9)=\left[\begin{array}{ll}
1 & 0 \\
0 & 1
\end{array}\right] \quad A(10)=\left[\begin{array}{ll}
0 & 1 \\
0 & 1
\end{array}\right] \quad A(12)=\left[\begin{array}{ll}
0 & 0 \\
1 & 1
\end{array}\right] \quad A(11)=\left[\begin{array}{ll}
1 & 1 \\
0 & 1
\end{array}\right] \\
& A(13)=\left[\begin{array}{ll}
1 & 0 \\
1 & 1
\end{array}\right] \quad A(15)=\left[\begin{array}{ll}
1 & 1 \\
1 & 1
\end{array}\right]
\end{aligned}
$$

[Case, $n=3$ ] There exist zero patterns of fuzzy idempotent except the zero matrix as follows;

$$
\begin{array}{ccc}
A(1)=\left[\begin{array}{lll}
1 & 0 & 0 \\
0 & 0 & 0 \\
0 & 0 & 0
\end{array}\right] & A(16)=\left[\begin{array}{lll}
0 & 0 & 0 \\
0 & 1 & 0 \\
0 & 0 & 0
\end{array}\right] & A(256)=\left[\begin{array}{lll}
0 & 0 & 0 \\
0 & 0 & 0 \\
0 & 0 & 1
\end{array}\right] \\
A(3)=\left[\begin{array}{lll}
1 & 1 & 0 \\
0 & 0 & 0 \\
0 & 0 & 0
\end{array}\right] & A(5)=\left[\begin{array}{lll}
1 & 0 & 1 \\
0 & 0 & 0 \\
0 & 0 & 0
\end{array}\right] & A(9)=\left[\begin{array}{lll}
1 & 0 & 0 \\
1 & 0 & 0 \\
0 & 0 & 0
\end{array}\right]
\end{array}
$$




$$
\begin{aligned}
& A(17)=\left[\begin{array}{lll}
1 & 0 & 0 \\
0 & 1 & 0 \\
0 & 0 & 0
\end{array}\right] \quad A(18)=\left[\begin{array}{lll}
0 & 1 & 0 \\
0 & 1 & 0 \\
0 & 0 & 0
\end{array}\right] \quad A(24)=\left[\begin{array}{lll}
0 & 0 & 0 \\
1 & 1 & 0 \\
0 & 0 & 0
\end{array}\right] \\
& A(48)=\left[\begin{array}{lll}
0 & 0 & 0 \\
0 & 1 & 1 \\
0 & 0 & 0
\end{array}\right] \quad A(65)=\left[\begin{array}{lll}
1 & 0 & 0 \\
0 & 0 & 0 \\
1 & 0 & 0
\end{array}\right] \quad A(144)=\left[\begin{array}{lll}
0 & 0 & 0 \\
0 & 1 & 0 \\
0 & 1 & 0
\end{array}\right] \\
& A(257)=\left[\begin{array}{lll}
1 & 0 & 0 \\
0 & 0 & 0 \\
0 & 0 & 1
\end{array}\right] \quad A(260)=\left[\begin{array}{lll}
0 & 0 & 1 \\
0 & 0 & 0 \\
0 & 0 & 1
\end{array}\right] \quad A(272)=\left[\begin{array}{lll}
0 & 0 & 0 \\
0 & 1 & 0 \\
0 & 0 & 1
\end{array}\right] \\
& A(288)=\left[\begin{array}{lll}
0 & 0 & 0 \\
0 & 0 & 1 \\
0 & 0 & 1
\end{array}\right] \quad A(320)=\left[\begin{array}{lll}
0 & 0 & 0 \\
0 & 0 & 0 \\
1 & 0 & 1
\end{array}\right] \quad A(384)=\left[\begin{array}{lll}
0 & 0 & 0 \\
0 & 0 & 0 \\
0 & 1 & 1
\end{array}\right] \\
& A(7)=\left[\begin{array}{lll}
1 & 1 & 1 \\
0 & 0 & 0 \\
0 & 0 & 0
\end{array}\right] \quad A(19)=\left[\begin{array}{lll}
1 & 1 & 0 \\
0 & 1 & 0 \\
0 & 0 & 0
\end{array}\right] \quad A(21)=\left[\begin{array}{lll}
1 & 0 & 1 \\
0 & 1 & 0 \\
0 & 0 & 0
\end{array}\right] \\
& A(25)=\left[\begin{array}{lll}
1 & 0 & 0 \\
1 & 1 & 0 \\
0 & 0 & 0
\end{array}\right] \quad A(49)=\left[\begin{array}{lll}
1 & 0 & 0 \\
0 & 1 & 1 \\
0 & 0 & 0
\end{array}\right] \quad A(56)=\left[\begin{array}{lll}
0 & 0 & 0 \\
1 & 1 & 1 \\
0 & 0 & 0
\end{array}\right] \\
& A(73)=\left[\begin{array}{lll}
1 & 0 & 0 \\
1 & 0 & 0 \\
1 & 0 & 0
\end{array}\right] \quad A(81)=\left[\begin{array}{lll}
1 & 0 & 0 \\
0 & 1 & 0 \\
1 & 0 & 0
\end{array}\right] \quad A(145)=\left[\begin{array}{lll}
1 & 0 & 0 \\
0 & 1 & 0 \\
0 & 1 & 0
\end{array}\right] \\
& A(146)=\left[\begin{array}{lll}
0 & 1 & 0 \\
0 & 1 & 0 \\
0 & 1 & 0
\end{array}\right] \quad A(259)=\left[\begin{array}{lll}
1 & 1 & 0 \\
0 & 0 & 0 \\
0 & 0 & 1
\end{array}\right] \quad A(261)=\left[\begin{array}{lll}
1 & 0 & 1 \\
0 & 0 & 0 \\
0 & 0 & 1
\end{array}\right] \\
& A(265)=\left[\begin{array}{lll}
1 & 0 & 0 \\
1 & 0 & 0 \\
0 & 0 & 1
\end{array}\right] \quad A(273)=\left[\begin{array}{lll}
1 & 0 & 0 \\
0 & 1 & 0 \\
0 & 0 & 1
\end{array}\right] \quad A(274)=\left[\begin{array}{lll}
0 & 1 & 0 \\
0 & 1 & 0 \\
0 & 0 & 1
\end{array}\right] \\
& A(276)=\left[\begin{array}{lll}
0 & 0 & 1 \\
0 & 1 & 0 \\
0 & 0 & 1
\end{array}\right] \quad A(280)=\left[\begin{array}{lll}
0 & 0 & 0 \\
1 & 1 & 0 \\
0 & 0 & 1
\end{array}\right] \quad A(289)=\left[\begin{array}{lll}
1 & 0 & 0 \\
0 & 0 & 1 \\
0 & 0 & 1
\end{array}\right] \\
& A(292)=\left[\begin{array}{lll}
0 & 0 & 1 \\
0 & 0 & 1 \\
0 & 0 & 1
\end{array}\right] \quad A(304)=\left[\begin{array}{lll}
0 & 0 & 0 \\
0 & 1 & 1 \\
0 & 0 & 1
\end{array}\right] \quad A(321)=\left[\begin{array}{lll}
1 & 0 & 0 \\
0 & 0 & 0 \\
0 & 0 & 1
\end{array}\right] \\
& A(336)=\left[\begin{array}{lll}
0 & 0 & 0 \\
0 & 1 & 0 \\
1 & 0 & 1
\end{array}\right] \quad A(385)=\left[\begin{array}{lll}
1 & 0 & 0 \\
0 & 0 & 0 \\
0 & 1 & 1
\end{array}\right] \quad A(400)=\left[\begin{array}{lll}
0 & 0 & 0 \\
0 & 1 & 0 \\
0 & 1 & 1
\end{array}\right]
\end{aligned}
$$




$$
\begin{aligned}
& A(448)=\left[\begin{array}{lll}
0 & 0 & 0 \\
0 & 0 & 0 \\
1 & 1 & 1
\end{array}\right] \quad A(23)=\left[\begin{array}{lll}
1 & 1 & 1 \\
0 & 1 & 0 \\
0 & 0 & 0
\end{array}\right] \quad A(27)=\left[\begin{array}{lll}
1 & 1 & 0 \\
1 & 1 & 0 \\
0 & 0 & 0
\end{array}\right] \\
& A(45)=\left[\begin{array}{lll}
1 & 0 & 1 \\
1 & 0 & 1 \\
0 & 0 & 0
\end{array}\right] \quad A(53)=\left[\begin{array}{lll}
1 & 0 & 1 \\
0 & 1 & 1 \\
0 & 0 & 0
\end{array}\right] \quad A(54)=\left[\begin{array}{lll}
0 & 1 & 1 \\
0 & 1 & 1 \\
0 & 0 & 0
\end{array}\right] \\
& A(57)=\left[\begin{array}{lll}
1 & 0 & 0 \\
1 & 1 & 1 \\
0 & 0 & 0
\end{array}\right] \quad A(89)=\left[\begin{array}{lll}
1 & 0 & 0 \\
1 & 1 & 0 \\
1 & 0 & 0
\end{array}\right] \quad A(147)=\left[\begin{array}{lll}
1 & 1 & 0 \\
0 & 1 & 0 \\
0 & 1 & 0
\end{array}\right] \\
& A(195)=\left[\begin{array}{lll}
1 & 1 & 0 \\
0 & 0 & 0 \\
1 & 1 & 0
\end{array}\right] \quad A(209)=\left[\begin{array}{lll}
1 & 0 & 0 \\
0 & 1 & 0 \\
1 & 1 & 0
\end{array}\right] \quad A(216)=\left[\begin{array}{lll}
0 & 0 & 0 \\
1 & 1 & 0 \\
1 & 1 & 0
\end{array}\right] \\
& A(263)=\left[\begin{array}{lll}
1 & 1 & 1 \\
0 & 0 & 0 \\
0 & 0 & 1
\end{array}\right] \quad A(275)=\left[\begin{array}{lll}
1 & 1 & 0 \\
0 & 1 & 0 \\
0 & 0 & 1
\end{array}\right] \quad A(277)=\left[\begin{array}{lll}
1 & 0 & 1 \\
0 & 1 & 0 \\
0 & 0 & 1
\end{array}\right] \\
& A(278)=\left[\begin{array}{lll}
0 & 1 & 1 \\
0 & 1 & 0 \\
0 & 0 & 1
\end{array}\right] \quad A(281)=\left[\begin{array}{lll}
1 & 0 & 0 \\
1 & 1 & 0 \\
0 & 0 & 1
\end{array}\right] \quad A(293)=\left[\begin{array}{lll}
1 & 0 & 1 \\
0 & 0 & 1 \\
0 & 0 & 1
\end{array}\right] \\
& A(297)=\left[\begin{array}{lll}
1 & 0 & 0 \\
1 & 0 & 1 \\
0 & 0 & 1
\end{array}\right] \quad A(305)=\left[\begin{array}{lll}
1 & 0 & 0 \\
0 & 1 & 1 \\
0 & 0 & 1
\end{array}\right] \quad A(308)=\left[\begin{array}{lll}
0 & 0 & 1 \\
0 & 1 & 1 \\
0 & 0 & 1
\end{array}\right] \\
& A(312)=\left[\begin{array}{lll}
0 & 0 & 0 \\
1 & 1 & 1 \\
0 & 0 & 1
\end{array}\right] \quad A(325)=\left[\begin{array}{lll}
1 & 0 & 1 \\
0 & 0 & 0 \\
1 & 0 & 1
\end{array}\right] \quad A(329)=\left[\begin{array}{lll}
1 & 0 & 0 \\
1 & 0 & 0 \\
1 & 0 & 1
\end{array}\right] \\
& A(337)=\left[\begin{array}{lll}
1 & 0 & 0 \\
0 & 1 & 0 \\
1 & 0 & 1
\end{array}\right] \quad A(344)=\left[\begin{array}{lll}
0 & 0 & 0 \\
1 & 1 & 0 \\
1 & 0 & 1
\end{array}\right] \quad A(360)=\left[\begin{array}{lll}
0 & 0 & 0 \\
1 & 0 & 1 \\
1 & 0 & 1
\end{array}\right] \\
& A(387)=\left[\begin{array}{lll}
1 & 1 & 0 \\
0 & 0 & 0 \\
0 & 1 & 1
\end{array}\right] \quad A(390)=\left[\begin{array}{lll}
0 & 1 & 1 \\
0 & 0 & 0 \\
0 & 1 & 1
\end{array}\right] \quad A(401)=\left[\begin{array}{lll}
1 & 0 & 0 \\
0 & 1 & 0 \\
0 & 1 & 1
\end{array}\right] \\
& A(402)=\left[\begin{array}{lll}
0 & 1 & 0 \\
0 & 1 & 0 \\
0 & 1 & 1
\end{array}\right] \quad A(432)=\left[\begin{array}{lll}
0 & 0 & 0 \\
0 & 1 & 1 \\
0 & 1 & 1
\end{array}\right] \quad A(449)=\left[\begin{array}{lll}
1 & 0 & 0 \\
0 & 0 & 0 \\
1 & 1 & 1
\end{array}\right] \\
& A(464)=\left[\begin{array}{lll}
0 & 0 & 0 \\
0 & 1 & 0 \\
1 & 1 & 1
\end{array}\right] \quad A(55)=\left[\begin{array}{lll}
1 & 1 & 1 \\
0 & 1 & 1 \\
0 & 0 & 0
\end{array}\right] \quad A(61)=\left[\begin{array}{lll}
1 & 0 & 1 \\
1 & 1 & 1 \\
0 & 0 & 0
\end{array}\right]
\end{aligned}
$$




$$
\begin{aligned}
& A(121)=\left[\begin{array}{lll}
1 & 0 & 0 \\
1 & 1 & 1 \\
1 & 0 & 0
\end{array}\right] \quad A(151)=\left[\begin{array}{lll}
1 & 1 & 1 \\
0 & 1 & 0 \\
0 & 1 & 0
\end{array}\right] \quad A(211)=\left[\begin{array}{lll}
1 & 1 & 0 \\
0 & 1 & 0 \\
1 & 1 & 0
\end{array}\right] \\
& A(217)=\left[\begin{array}{lll}
1 & 0 & 0 \\
1 & 1 & 0 \\
1 & 1 & 0
\end{array}\right] \quad A(279)=\left[\begin{array}{lll}
1 & 1 & 1 \\
0 & 1 & 0 \\
0 & 0 & 1
\end{array}\right] \quad A(283)=\left[\begin{array}{lll}
1 & 1 & 0 \\
1 & 1 & 0 \\
0 & 0 & 1
\end{array}\right] \\
& A(295)=\left[\begin{array}{lll}
1 & 1 & 1 \\
0 & 0 & 1 \\
0 & 0 & 1
\end{array}\right] \quad A(301)=\left[\begin{array}{lll}
1 & 0 & 1 \\
1 & 0 & 1 \\
0 & 0 & 1
\end{array}\right] \quad A(309)=\left[\begin{array}{lll}
1 & 0 & 1 \\
0 & 1 & 1 \\
0 & 0 & 1
\end{array}\right] \\
& A(310)=\left[\begin{array}{lll}
0 & 1 & 1 \\
0 & 1 & 1 \\
0 & 0 & 1
\end{array}\right] \quad A(313)=\left[\begin{array}{lll}
1 & 0 & 0 \\
1 & 1 & 1 \\
0 & 0 & 1
\end{array}\right] \quad A(316)=\left[\begin{array}{lll}
0 & 0 & 1 \\
1 & 1 & 1 \\
0 & 0 & 1
\end{array}\right] \\
& A(341)=\left[\begin{array}{lll}
1 & 0 & 1 \\
0 & 1 & 0 \\
1 & 0 & 1
\end{array}\right] \quad A(345)=\left[\begin{array}{lll}
1 & 0 & 0 \\
1 & 1 & 0 \\
1 & 0 & 1
\end{array}\right] \quad A(361)=\left[\begin{array}{lll}
1 & 0 & 0 \\
1 & 0 & 1 \\
1 & 0 & 1
\end{array}\right] \\
& A(376)=\left[\begin{array}{lll}
0 & 0 & 0 \\
1 & 1 & 1 \\
1 & 0 & 1
\end{array}\right] \quad A(391)=\left[\begin{array}{lll}
1 & 1 & 1 \\
0 & 0 & 0 \\
0 & 1 & 1
\end{array}\right] \quad A(403)=\left[\begin{array}{lll}
1 & 1 & 0 \\
0 & 1 & 0 \\
0 & 1 & 1
\end{array}\right] \\
& A(406)=\left[\begin{array}{lll}
0 & 1 & 1 \\
0 & 1 & 0 \\
0 & 1 & 1
\end{array}\right] \quad A(433)=\left[\begin{array}{lll}
1 & 0 & 0 \\
0 & 1 & 1 \\
0 & 1 & 1
\end{array}\right] \quad A(451)=\left[\begin{array}{lll}
1 & 1 & 0 \\
0 & 0 & 0 \\
1 & 1 & 1
\end{array}\right] \\
& A(457)=\left[\begin{array}{lll}
1 & 0 & 0 \\
1 & 0 & 0 \\
1 & 1 & 1
\end{array}\right] \quad A(465)=\left[\begin{array}{lll}
1 & 0 & 0 \\
0 & 1 & 0 \\
1 & 1 & 1
\end{array}\right] \quad A(466)=\left[\begin{array}{lll}
0 & 1 & 0 \\
0 & 1 & 0 \\
1 & 1 & 1
\end{array}\right] \\
& A(472)=\left[\begin{array}{lll}
0 & 0 & 0 \\
1 & 1 & 0 \\
1 & 1 & 1
\end{array}\right] \quad A(63)=\left[\begin{array}{lll}
1 & 1 & 1 \\
1 & 1 & 1 \\
0 & 0 & 0
\end{array}\right] \quad A(219)=\left[\begin{array}{lll}
1 & 1 & 0 \\
1 & 1 & 0 \\
1 & 1 & 0
\end{array}\right] \\
& A(311)=\left[\begin{array}{lll}
1 & 1 & 1 \\
0 & 1 & 1 \\
0 & 0 & 1
\end{array}\right] \quad A(317)=\left[\begin{array}{lll}
1 & 0 & 1 \\
1 & 1 & 1 \\
0 & 0 & 1
\end{array}\right] \quad A(365)=\left[\begin{array}{lll}
1 & 0 & 1 \\
1 & 0 & 1 \\
1 & 0 & 1
\end{array}\right] \\
& A(377)=\left[\begin{array}{lll}
1 & 0 & 0 \\
1 & 1 & 1 \\
1 & 0 & 1
\end{array}\right] \quad A(407)=\left[\begin{array}{lll}
1 & 1 & 1 \\
0 & 1 & 0 \\
0 & 1 & 1
\end{array}\right] \quad A(438)=\left[\begin{array}{lll}
0 & 1 & 1 \\
0 & 1 & 1 \\
0 & 1 & 1
\end{array}\right] \\
& A(455)=\left[\begin{array}{lll}
1 & 1 & 1 \\
0 & 0 & 0 \\
1 & 1 & 1
\end{array}\right] \quad A(467)=\left[\begin{array}{lll}
1 & 1 & 0 \\
0 & 1 & 0 \\
1 & 1 & 1
\end{array}\right] \quad A(473)=\left[\begin{array}{lll}
1 & 0 & 0 \\
1 & 1 & 0 \\
1 & 1 & 1
\end{array}\right]
\end{aligned}
$$




$$
\begin{gathered}
A(504)=\left[\begin{array}{lll}
0 & 0 & 0 \\
1 & 1 & 1 \\
1 & 1 & 1
\end{array}\right] \quad A(319)=\left[\begin{array}{lll}
1 & 1 & 1 \\
1 & 1 & 1 \\
0 & 0 & 1
\end{array}\right] \quad A(381)=\left[\begin{array}{lll}
1 & 0 & 1 \\
1 & 1 & 1 \\
1 & 0 & 1
\end{array}\right] \\
A(439)=\left[\begin{array}{lll}
1 & 1 & 1 \\
0 & 1 & 1 \\
0 & 1 & 1
\end{array}\right] \quad A(471)=\left[\begin{array}{lll}
1 & 1 & 1 \\
0 & 1 & 0 \\
1 & 1 & 1
\end{array}\right] \quad A(475)=\left[\begin{array}{lll}
1 & 1 & 0 \\
1 & 1 & 0 \\
1 & 1 & 1
\end{array}\right] \\
A(505)=\left[\begin{array}{lll}
1 & 0 & 0 \\
1 & 1 & 1 \\
1 & 1 & 1
\end{array}\right] \quad A(511)=\left[\begin{array}{lll}
1 & 1 & 1 \\
1 & 1 & 1 \\
1 & 1 & 1
\end{array}\right]
\end{gathered}
$$

Remark 4.1. There exists many zero patterns of fuzzy idempotent for dimension 4 and 5, we give the zero patterns that the form is $A_{n}(p)=$ $q$ where each $n, p$ and $q$ means dimension, the number of 1 's in a zero pattern $A$ and the number of zero patterns satisfied $n$ and $p$.

[Case, $n=4]$ There are all zero patterns of fuzzy idempotent with the form $A_{n}(p)=q$ as follows;

$$
\begin{aligned}
& A_{4}(0)=1, \quad A_{4}(1)=4, \quad A_{4}(2)=30, \quad A_{4}(3)=88, \\
& A_{4}(4)=231, \quad A_{4}(5)=360, \quad A_{4}(6)=494, \quad A_{4}(7)=428, \\
& A_{4}(8)=357, \quad A_{4}(9)=196, \quad A_{4}(10)=112, \quad A_{4}(11)=36, \\
& A_{4}(12)=14, \quad A_{4}(13)=8, \quad A_{4}(16)=1
\end{aligned}
$$

Therefore, all zero patterns of fuzzy idempotent that is dimension 4 exists 2360's.

[Case, $n=5]$ There are all zero patterns of fuzzy idempotent with the form $A_{n}(p)=q$ as follows;

$$
\begin{aligned}
& A_{5}(0)=1, \quad A_{5}(1)=5, \quad A_{5}(2)=50, \quad A_{5}(3)=210, \quad A_{5}(4)=835, \\
& A_{5}(5)=2161, \quad A_{5}(6)=4810, \quad A_{5}(7)=7770, \quad A_{5}(8)=10635, \\
& A_{5}(9)=11815, \quad A_{5}(10)=11200, \quad A_{5}(11)=9070, \quad A_{5}(12)=6330, \\
& A_{5}(13)=4040, \quad A_{5}(14)=2220, \quad A_{5}(15)=1140, \quad A_{5}(16)=395, \\
& A_{5}(17)=235, \quad A_{5}(18)=60, \quad A_{5}(19)=20, \quad A_{5}(20)=10, \\
& A_{5}(21)=10, \quad A_{5}(25)=1
\end{aligned}
$$

Therefore, all zero patterns of fuzzy idempotent that is dimension 5 exists 703023's. 


\section{References}

[1] K. H. Kim and F. W. Roush, Generalized fuzzy matrices, Fuzzy Sets and Systems 4 (1980), 293-315.

[2] K. H. Kim and F. W. Roush, Idempotent fuzzy matrices, Seminar Report, Alabama State Univ., Montgomery, AL (1981).

[3] H. Hashimoto, Canonical form of a transitive fuzzy matrix, Fuzzy Sets and Systems 11 (1983), 157-162

[4] H. Schneider The influence of the marked reduced graph of a nonnegative matrix on the Jordan form and on related properties, Linear Algebra Appl. 84 (1986), $161-189$

[5] H. Y. Lee and S. W. Park The Boolean idempotent matrices, Journal of Applied Math. and copmuting. 15 (2004), 475-484

Se Won Park

Department of Liberal arts and Science, Shingyeong University, 400 Namyang-ro Hwaseong-si, Kyonggi-do, KOREA. 445-741

E-mail : sewpark1079@naver.com

Chul Kang

Department of Applied Mathematics, Hankyong National University, 327 Chungang-no Anseong-si, Kyonggi-do, KOREA. 456-749

E-mail : stat@hknu.ac.kr 